\title{
Biosimilars: the impact of their heterogeneity on regulatory approval
}

The development of first-generation recombinant therapeutic proteins through genetic engineering and the production of therapeutically effective monoclonal antibodies (mAbs) are considered major achievements of modern biotechnology. Today, more than 20 years since the first such biologic drugs were approved, a growing number of patents for such products are expiring. The possibilities for manufacturing and marketing second-generation generic biologics are therefore attracting increasing attention ${ }^{1}$, and the demand for such products at reduced prices is higher than ever.

However, biologics produced by various systems (animal cells, insect cells, prokaryotes, plants, yeast or whole organisms) are inherently variable and heterogeneous in many respects (such as starting materials, host, molecular structure and manufacturing process), which has the potential to translate into significant differences in potency, pharmacokinetics and clinical safety and efficacy. The conventional regulatory framework applied for the development and market authorisation of generic small-molecule drugs (typically made by chemical synthesis, and readily characterizable by standard analytical techniques) is therefore no longer suitable for biologics.

With this in mind, the European Union has established a new system to deal exclusively with biologics (for example, mAbs or recombinant proteins) that share similar action (now called biosimilar medicinal products). The recently established regulatory framework (see Guideline for Similar Biological Medicinal products issued by the European Medicines Agency (EMEA) ${ }^{2,3}$ ) is a major step in the regulatory approval of high-quality biosimilars. This market authorisation approval (MAA) process, however, is quite different from the process currently applied by the Food and Drug Administration (FDA) in the United States, in which biologics and/or biotech products of similar action are approved on a case-by-case basis. This is the process, at least until the FDA also introduces a new regulatory process for follow-on biologics.

The guidelines on biosimilar medicinal products issued by the EMEA call for full comparability studies between the original product and the new (to be approved) biosimilar product on both preclinical and clinical levels. The major questions that arise are whether such comparability studies are possible in every case or whether difficulties in carrying out such requirements successfully will affect the MAA process. Larger proteins are complex, as they can exist in different isoforms and undergo post-translational modifications (such as glycosylation, methylation, acetylation and phosphorylation) that can affect their physicochemical behaviour (hydrophobicity/hydrophilicity, solubility, folding and aggregation) and most importantly, their pharmacological action on targeted tissues. Furthermore, biosimilars might be produced by differing manufacturing processes using diverse host systems with 
different capacities to post-translationally modify proteins, increasing the potential for significant differences with the original product. Such heterogeneity in biosimilars might affect the quality of the active substance as well as the drug product in terms of biological activity, stability, pharmacokinetics, immunogenicity and clinical efficacy. It is reasonable then to ask whether the extent of such heterogeneity will affect the successful outcome of full comparability studies, and in turn the MAA.

So, as successful full comparability studies are a prerequisite, each biosimilar product must undergo vigorous quality evaluation, which can now be done efficiently with currently applied process analytical technologies (including full-length peptide mapping, MALDI TOF (matrix-assisted laser desorption/ionization time of flight) mass spectroscopy, circular dichroism and analysis of glycosylation patterns). There is now evidence to indicate that the glycosylation pattern of biosimilars determines their immunogenicity and affects the biological activity of mAbs ${ }^{4-6}$. Emphasis must also be given to the study of the physical state and stability of the active substance, as several biosimilars are produced in bacteria in inclusion bodies, and then dissolved and refolded under different conditions. Detailed analysis of biosimilar products can then provide a clearer picture of the molecular structure and function of both the active substance and the final product. Evaluation of biosimilars in suitable cellculture and animal model systems is also crucial, as comparison with the reference product can identify similarities as well as major differences in potency. Last, comparability studies at the clinical level can confirm the pharmacological potency expected as well as unique adverse reactions (such as immunogenic responses).

The policy of the EMEA to provide scientific advice through the Biologics Working Party (BWP) of the Committee for Medicinal Products for Human Use (CHMP) to the developers of biosimilar products has important implications for the biopharma industry. This strategy, together with the guidelines on biosimilars, will facilitate the process of developing and approving high-quality second-generation biologics without delays.

Asterios S. Tsiftsoglou

Professor of Pharmacology, Aristotle University of Thessaloniki, Thessaloniki, Greece, and Core Member of Biologics Working Party (BWP) and European Medicines Agency (EMEA), London, UK.

e-mail: tsifepharm.auth.gr

1. Belsey, M. J. et al. Biosimilars: initial excitement gives way to reality. Nature Rev. Drug Discov. 5, 535536 (2006).

2. EMEA/CHMP. Guideline on Similar Biological Medicinal Products Containing Biotechnology-Derived Proteins as Active Substance: Nonclinical and Clinical Issues[online], 
<http.//www.emea.eu.int/pdfs/hman biosimilar/4283205en.pdf (2005).

3. EMEA/CHMP. Guideline on Similar Biological Medicinal Products Containing Biotechnology-Derived Proteins as Active Substance: Quality Issues [online], <http://www.emea.eu.int?pdfs/human biosimilar/4934805en.pdy $(2005)$.

4. Royston, J. Glycosylation of recombinant antibody therapeutics. Biotechnol. Prof. 21, 1116 (2005)

5. Burton D. R \& Dwek, R. A. Sugar determines antibody activity. Science 313, 627628 (2006).

6. Kaneko, Y., Ninumerrjahn, F. \& Ravetch, J. V. Anti inflammatory activity of immunoglobulin G resulting from FC sialyation. Science 313, 670673 (2006). 